\title{
Lipidomic signatures of post-hepatectomy liver failure using porcine hepatectomy models
}

\author{
Hye-Sung Jo ${ }^{1 \#}$, Hae A. Kim ${ }^{2 \#}$, Jong Cheol Lee ${ }^{2}$, Kyung Chul Yoon ${ }^{1}$, Young-In Yoon ${ }^{3}$, Yoon Young Choi ${ }^{4}$, \\ Jin-I Seok ${ }^{4}$, Myeong Hee Moon ${ }^{2}$, Dong-Sik Kim ${ }^{1}$ \\ ${ }^{1}$ Department of Surgery, Korea University College of Medicine, Seoul, Korea; ${ }^{2}$ Department of Chemistry, Yonsei University, Seoul, Korea; \\ ${ }^{3}$ Department of Surgery, University of Ulsan College of Medicine, Seoul, Korea; ${ }^{4}$ Department of Biomedical Science, Korea University College of \\ Medicine Graduate School, Seoul, Korea \\ Contributions: (I) Conception and design: HS Jo, HA Kim, DS Kim, MH Moon; (II) Administrative support: YY Choi, JI Seok; (III) Provision of \\ study materials or patients: HS Jo, KC Yoon, YI Yoon; (IV) Collection and assembly of data: HA Kim, JC Lee, YY Choi, JI Seok; (V) Data analysis \\ and interpretation: All authors; (VI) Manuscript writing: All authors; (VII) Final approval of manuscript: All authors. \\ \#These authors contributed equally to this work. \\ Correspondence to: Dong-Sik Kim, MD, PhD. Division of HBP Surgery and Liver Transplantation, Department of Surgery, Korea University College \\ of Medicine, Seoul, 02841, Korea. Email: kimds1@korea.ac.kr; Myeong Hee Moon, PhD. Department of Chemistry, Yonsei University, Seoul 03722, \\ Korea. Email: mhmoon@yonsei.ac.kr.
}

Background: Clinical diagnosis of post-hepatectomy liver failure (PHLF) can only be made on or after the 5 th postoperative day. Biomarker for early diagnosis is considered as a critical unmet need.

Methods: Twenty domestic female crossbreed (Yorkshire-landrace and duroc) pigs underwent sham operation ( $\mathrm{n}=6), 70 \%(\mathrm{n}=7)$ and 90\% ( $=7$ ) partial hepatectomy $(\mathrm{PH})$. A comprehensive lipidomic analysis was conducted using sera collected at pre-operation (PO), 14, 30, and 48 h after PH using nanoflow ultrahigh performance liquid chromatography-electrospray ionization-tandem mass spectrometry.

Results: Of the 184 quantified lipids, 14 lipids showed significant differences between the two resection groups starting at $30 \mathrm{~h}$ after surgery. Four phosphatidylcholine (PC) plasmalogen species (P-16:0/16:0, P-18:0/18:2, P-18:0/20:4, and P-18:0/22:6) and PC 32:2 significantly increased in the 90\% PH group while these returned to PO level after $30 \mathrm{~h}$ in the $70 \% \mathrm{PH}$ group, presumably implying the failure markers. In contrast, eight triacylglycerol (TG) species (40:0, 42:1, 42:0, 44:1, 44:2, 46:1, 46:2, and 48:3) and sphingomyelin d18:1/20:0 showed an opposite trend, wherein they significantly decreased in the $90 \% \mathrm{PH}$ group while these in the $70 \% \mathrm{PH}$ group were abruptly increased until $30 \mathrm{~h}$ but returned to near PO levels at $48 \mathrm{~h}$, implying the recovery markers. Same trends could also be observed in the level of whole lipid classes of PC plasmalogens and TGs, in addition to selected individual lipid species.

Conclusions: Characteristic lipidomic signatures of PHLF could be identified using large animal models. These candidates have a potential to serve as a tool for early diagnosis and may open new paths to the study to overcome PHLF.

Keywords: Biomarkers; liver failure; liver regeneration; lipidomics

Submitted Apr 29, 2020. Accepted for publication Sep 18, 2020.

doi: 10.21037/atm-20-3596

View this article at: http://dx.doi.org/10.21037/atm-20-3596

\section{Introduction}

Due to the extraordinary regeneration capability of the liver, liver resection has been used as an effective and safe therapy to treat tumors and is increasingly being used to cope with a variety of benign and malignant conditions $(1,2)$. However, post-hepatectomy liver failure (PHLF) occurs with an incidence rate of $0.7-34 \%$ and is a predominant source of morbidity and mortality after liver resection (3-6). Therefore, 
prevention, early diagnosis, and treatment of PHLF are important unmet needs in the treatment of liver diseases. PHLF is a postoperatively acquired deterioration in the ability of the liver to maintain its synthetic, excretory, and detoxifying functions, thus failing to meet one's metabolic demands. It is known to be associated with the remnant liver volume as well as functional quality of liver parenchyma (1). The dominant underlying pathophysiology of PHLF is considered to be increased portal flow to reduced volume of liver parenchyma accompanied by the elevation of portal pressure, which leads to shear stress and injury to hepatic sinusoidal endothelium $(7,8)$. Such injury process is initiated early after the liver resection and is sustained even before the clinical signs become evident.

Currently, the definition provided by the International Study Group of Liver Surgery (2) and the "50-50" criteria (9) are the most widely used methods for clinical diagnosis of PHLF; both criteria include serum bilirubin and prothrombin time. However, by definition, diagnosis can be made only at or after postoperative day 5, when damage to the remnant liver is already profound. Therefore, establishment of prognostic molecular markers enabling earlier diagnosis could be the most important step to develop strategies to overcome PHLF.

Lipids play important roles in various biological processes $(10,11)$, and the liver plays important roles in lipid metabolism. Lipids are synthesized in the liver and exported to and from the rest of the body by lipoproteins (12). Lipid metabolism can be significantly altered during liver disease (13) or after liver resection (14-19), suggesting their potential to be used as biomarkers in the setting of PHLF. However, lipid profiles during PHLF have not been thoroughly examined. Considering the difficulties in the study using humanderived samples from this grave condition, a systematic lipidomic analysis at the molecular level using well-designed animal model is expected to provide an insight into the relation of PHLF with liver function and regeneration.

In this study, a comprehensive lipidomic analysis of pig serum samples obtained after extensive hepatectomy was carried out using nanoflow ultrahigh performance liquid chromatography with electrospray ionization mass spectrometry (nUHPLC-ESI-MS/MS), which could characterize various isoforms of lipid with the limit of detection (LOD) at low femtomole levels $(20,21)$. The present study aimed to compare the time-dependent changes in the individual lipid levels after extensive hepatectomy to select potential prognostic lipid markers that can be utilized to predict the early stage of PHLF.
We present the following article in accordance with the ARRIVE reporting checklist (available at http://dx.doi. org/10.21037/atm-20-3596).

\section{Methods}

\section{Animals and experimental groups}

Twenty domestic female crossbreed (Yorkshire-landrace and duroc) pigs were employed in this study. The median age was 3 (range, 2-3) months, and body weight was $35.9(32.8-43.0) \mathrm{kg}$. The animals were housed at $22 \pm 1{ }^{\circ} \mathrm{C}$ with a 12-h light/dark cycle with free access to water and food. Pigs were not fed for 8 hours before the operation. Pigs were divided into three groups: the $70 \%$ partial hepatectomy $(\mathrm{PH})$ group $(\mathrm{n}=7), 90 \% \mathrm{PH}$ group $(\mathrm{n}=7)$, and sham group $(n=6)$ with the same open surgery except the liver resection. This study was approved by the Korea University Institutional Animal Care and Use Committee (IRB: KOREA-2017-0069-C3) and all animals were cared for in accordance with the National guidelines for ethical animal research.

\section{Anesthesia and liver resection}

All pigs were anesthetized in the following way. Initial sedation was carried out with an intramuscular injection of alfaxalone $(4 \mathrm{mg} / \mathrm{kg}$, Jurox Inc. North Kansas City, MO, USA), xylazine (1.5 mg/kg, Bayer Korea, Seoul, Korea), and azaperone ( $4 \mathrm{mg} / \mathrm{kg}$, SF Inc., Ansan, Korea). A $6.5 \mathrm{~mm}$ endotracheal tube was inserted and anesthesia was maintained using 2\% isoflurane (Hana pharm CO., LTD, Seoul, Korea) in oxygen titrated to a depth of anesthesia. Oxygen saturation and heart rate were monitored throughout the operation. Triple lumen central venous catheter (Cook medical LLC, Bloomington, IN, USA) was inserted via right jugular vein under direct visualization, fixed under the skin using tunneling method, and sutured with 2-0 nylon. This catheter remained in situ until sacrifice for blood sampling and intravenous fluid administration.

A midline laparotomy was performed extending from the xiphoid process to below the umbilicus. The liver was mobilized from ligamentous structures. The left lateral lobe, the left medial lobe, and the right medial lobe except the caudate area covering the inferior vena cava were resected for $70 \%$ hepatectomy. The second order or third order branch of the Glissonian pedicle was ligated to each lobe and parenchymal transection was performed using 
the Kelly crushing method along the ischemic line. The left and middle hepatic veins were suture-ligated, and the right hepatic vein trunk was preserved for the right lateral lobe. For $90 \%$ hepatectomy, two-third of the remaining right lateral lobe was resected with the same maneuver. Abdominal wall was closed after adequate hemostasis. For pigs in the sham group, the abdominal wall was closed without liver resection after incision and exposure. After the surgery, 5\% Hartmann dextrose (JW Pharmaceutical, Seoul, Korea) fluid was administered until sacrifice. In addition, ketoprofen ( $3 \mathrm{mg} / \mathrm{kg}$, Ubtech, Anyang, Korea) was injected subcutaneously once after surgery. Each pig could access the food and water ad libitum in the post-operative phase.

\section{Serum samples and biomedical analysis}

Serum samples from all the animals were collected before skin incision and at seven time points after the operation (1, $6,14,22,30,38$, and 48 h). Animals were sacrificed 48 hours after hepatectomy. The levels of aspartate aminotransferase (AST), alanine aminotransferase (ALT), total bilirubin (TBIL), and prothrombin time as international normalized ratio (PT-INR) were measured for each animal at different time points before and after the operation (Chemical analyzer AU5800, Beckmann coulter Inc., Brea, CA, USA and Blood Coagulation Diagnosis analyzer, Diagnostica Stago Inc., Parsippany, NJ, USA). A total of 80 serum samples (PO, 14, 30, and $80 \mathrm{~h}$ ) were used for lipid analysis. Serum samples were stored at $-80^{\circ} \mathrm{C}$ until lipid extraction.

\section{Materials and reagents for lipid analysis}

A total of 38 lipid standards were utilized to optimize nUHPLCESI-MS/MS run conditions: lysophosphatidylcholine (LPC) 16:0, 17:0, phosphatidylcholine (PC) 12:0/12:0, 13:0/13:0, 16:0/16:0, PC plasmalogen P-18:0/22:6, lysophosphatidylethanolamine (LPE) 18:0, 17:1, phosphatidylethanolamine (PE) 12:0/12:0, 17:0/17:0, PE plasmalogen P-18:0/18:1, P-18:0/22:6, lysophosphatidylglycerol (LPG) 18:0, 17:1, phosphatidylglycerol (PG) 14:0/14:0, 15:0/15:0, lysophosphatidylinositol (LPI) 20:4, 18:0, 17:1, phosphatidylinositol (PI) 16:0/18:1, 18:0/18:0, 12:0/13:0, lysophosphatidylserine (LPS) 18:1, lysophosphatidic acid (LPA) 14:0, 17:0, diacylglycerol (DG) 16:0/18:1, 17:0/17:0 D4, triacylglycerol (TG) 18:0/18:1/18:1, 17:0/17:1/17:0 D4, sphingomyelin (SM) d 18:1/16:0, d18:1/18:0, d18:1/17:0, ceramide (Cer) d18:1/14:0, d18:1/17:0, monohexosylceramide (HexCer) d18:1/16:0, d18:1/17:0, d18:1/24:0, and cardiolipin (CL) (14:0) 4 . Among these, 14 lipids with odd numbered acyl chain were used as internal standards (ISs) added to serum samples for quantitation of serum lipids. All lipid standards were purchased from Avanti Polar Lipids, Inc. (Alabaster, AL, USA). HPLC grade solvents were utilized for nUHPLC: $\mathrm{MeOH}, \mathrm{ACN}$, IPA, and $\mathrm{H}_{2} \mathrm{O}$ were purchased from J.T. baker (Phillipsburg, NJ, USA) and methyl-tert-butyl ether (MTBE), $\mathrm{CHCl}_{3}, \mathrm{NH}_{4} \mathrm{HCO}_{2}$, and $\mathrm{NH}_{4} \mathrm{OH}$ were purchased from Sigma-Aldrich (St. Louis, MO, USA). Fused silica capillaries with inner diameters of 20, 50, and $100 \mu \mathrm{m}$ (360 $\mu \mathrm{m}$ outer diameter for all) were purchased from Polymicro Technology (Phoenix, AZ, USA). For the column packing, $1.7 \mu \mathrm{m}$ ethylene bridged hybrid $(\mathrm{BEH})$ particles unpacked from ACQUITY UPLC ${ }^{\circledR}$ BEH C10 column $(2.1 \mathrm{~mm} \times 100 \mathrm{~mm})$ from Waters ${ }^{\mathrm{TM}}$ (Milford, MA, USA) and Watchers ${ }^{\circledR}$ ODS-P C-18 particles $(3 \mu \mathrm{m}$ and $100 \AA$ ) from Isu Industry Corp. (Seoul, Korea) were utilized.

\section{Lipid extraction and nUHPLC-ESI-MS/MS analysis}

Lipids were extracted using the two-stage extraction method with $\mathrm{MTBE} / \mathrm{CH}_{3} \mathrm{OH}$. For the lipid identification, a pooled serum sample was prepared for lipid extraction for each group by pooling the two serum samples [preoperation (PO) and $48 \mathrm{~h}$ ] in each group into a total volume of $100 \mu \mathrm{L}$. For targeted lipid quantitation, each sample was extracted individually. In both cases, $100 \mu \mathrm{L}$ of serum was initially dried with $\mathrm{N}_{2}$ gas using an Evatros Mini evaporator from Goojung Engineering Co., Ltd. (Seoul, Korea). A total of $300 \mu \mathrm{L} \mathrm{MeOH}$ was then added to the dried sample and it was subsequently placed in an ice bath for $10 \mathrm{~min}$. The dissolved serum was mixed with $1000 \mu \mathrm{L}$ MTBE and vortexed for an hour. After vortexing, $250 \mu \mathrm{L}$ of $\mathrm{H}_{2} \mathrm{O}$ was added to the mixture followed by vortexing for 10 $\mathrm{min}$ and centrifugation at $1000 \times g$ for $10 \mathrm{~min}$. The upper organic layer was transferred to another tube and $300 \mu \mathrm{L}$ MTBE was added to the remaining lower aqueous layer followed by tip sonication for $2 \mathrm{~min}$ and centrifugation at $1000 \times g$ for $10 \mathrm{~min}$. The resulting upper phase was then mixed with the previously collected organic phase. The final mixture was dried under $\mathrm{N}_{2}$ gas and the dried lipids were re-dissolved in $\mathrm{MeOH}: \mathrm{CHCl}_{3}(9: 1 \mathrm{v} / \mathrm{v})$ and stored at $-80{ }^{\circ} \mathrm{C}$. For nUHPLC-ESI-MS/MS analysis, the frozen lipid sample was diluted to final concentration of $5 \mu \mathrm{g} / \mu \mathrm{L}$ using $\mathrm{MeOH}: \mathrm{H}_{2} \mathrm{O}$ (8:2, v/v).

Two nUHPLC-ESI-MS/MS systems were employed 
in this study. For non-targeted lipid identification, pooled serum samples were analyzed by Thermo Fischer Scientific ${ }^{\mathrm{TM}}$ UltiMate $^{\mathrm{TM}} 3000$ RSLCnano System coupled with Thermo LTQ Velos ion trap mass spectrometer which was purchased from Thermo Fisher Scientific (Waltham, MA, USA). For the identification of lipid molecular structure, data-dependent collision induced dissociation experiments were accomplished during nUHPLCESI-MS/MS. Identified lipids were quantified by using nanoACQUITY UPLC system from Waters (Milford, MA, USA) coupled with TSQ Vantage triple-stage quadrupole mass spectrometer from Thermo Fischer Scientific. For data-dependent collision induced dissociation experiments, $40 \%$ normalized collision energy was utilized. Identification of the lipid molecular structures was achieved using LiPilot, a PC-based software in our laboratory (22).

\section{nUHPLC-ESI-MS/MS analysis of lipids}

The analytical column was prepared in our laboratory by packing $1.7 \mu \mathrm{m}$ BEH C18 beads in a silica capillary $(100 \mu \mathrm{m}$ inner diameter) of which one end of tube was pulled into a sharp needle by flame to be used as a direct emitter for ESI. Prior to column packing, the pulled tip was packed with $3 \mu \mathrm{m}$ Watchers ODS-P C18 particles to make selfassembled frit for $0.5 \mathrm{~cm}$. The column was connected with a capillary tube ( $50 \mu \mathrm{m}$ inner diameter) from the UHPLC pump via PEEK micro-cross, of which the remaining two ports were connected to a $\mathrm{Pt}$ wire for voltage supply and a pressure capillary (20 $\mu \mathrm{m}$ inner diameter) terminated with an on/off switching valve to split pump flow. The latter valve was closed during sample loading and open during separation so that the flow rate entering the column was adjusted to $300 \mathrm{~nL} / \mathrm{min}$.

Mobile phase solutions were $(9: 1, \mathrm{v} / \mathrm{v}) \mathrm{H} 2 \mathrm{O}: \mathrm{CH} 3 \mathrm{CN}$ for $\mathrm{A}$ and $(2: 2: 6, \mathrm{v} / \mathrm{v} / \mathrm{v}) \mathrm{CH}_{3} \mathrm{OH}: \mathrm{CH}_{3} \mathrm{CN}$ :isopropanol for $\mathrm{B}$, both were added with $0.05 \% \mathrm{NH}_{4} \mathrm{OH}$ and $5 \mathrm{mM}$ $\mathrm{NH}_{4} \mathrm{HCO}_{2}$ as a mixed ionization modifier. For non-targeted analysis, $2 \mu \mathrm{L}$ of lipid extract sample $(5 \mu \mathrm{g} / \mu \mathrm{L})$ was loaded with $99 \% \mathrm{~A}$ at a flow rate of $700 \mathrm{~nL} / \mathrm{min}$ for $10 \mathrm{~min}$. After loading, the pump flow rate was increased to $7.5 \mu \mathrm{L} / \mathrm{min}$ with the switching valve on so that the dwell time can be minimized with the splitting of flow entering the column at $300 \mathrm{~nL} / \mathrm{min}$. Gradient elution at positive ion mode began with $65 \%$ B to $85 \%$ B for $7 \mathrm{~min}$, further increased to $95 \%$ during $25 \mathrm{~min}$, and then ramped to $99 \%$ for $2 \mathrm{~min}$. After maintaining at $99 \% \mathrm{~B}$ for $10 \mathrm{~min}$, it was resumed to $1 \%$ B for column reconditioning. Gradient at negative ion mode began with $60 \%$ B by ramping to $70 \%$ for $5 \mathrm{~min}$., increasing to $95 \%$ for $30 \mathrm{~min}$., and further to $99 \%$ for $4 \mathrm{~min}$. After maintaining at $99 \%$ for $10 \mathrm{~min}$, it was resumed to $1 \%$ B. For lipid detection, a precursor MS scan was set to $\mathrm{m} / \mathrm{z} 300-1,500 \mathrm{amu}$ with $3.0 \mathrm{kV}$ of ESI voltage for both positive and negative ion modes.

For targeted quantification of lipids, $2 \mu \mathrm{L}$ of individual serum sample added with a mixture of odd-numbered standards (2 pmol each) was loaded to column with $100 \%$ $\mathrm{A}$ at a flow rate of $800 \mathrm{~nL} / \mathrm{min}$ for $10 \mathrm{~min}$. After loading, the flow rate was adjusted to $15 \mu \mathrm{L} / \mathrm{min}$ with the column flow rate at $300 \mathrm{~nL} / \mathrm{min}$. Gradient elution began with $55 \% \mathrm{~B}$ and it was increased to $90 \%$ for $10 \mathrm{~min}$, to $95 \%$ for $2 \mathrm{~min}$, and further to $100 \%$ for $8 \mathrm{~min}$. After maintaining at $100 \% \mathrm{~B}$ for $5 \mathrm{~min}$, it was resumed to $100 \% \mathrm{~A}$ for $5 \mathrm{~min}$. For quantification of lipids, selected reaction monitoring (SRM)-based analysis was made by scanning precursor ion and a selected product ion with specific CID energies in the polarity switching mode (alternating detection at positive and negative ion mode). Types of precursor ion and specific product ion for SRM quantification are listed in Table S1. While ESI voltage was fixed at $3 \mathrm{kV}$, collision energy was assigned differently depending on the lipid classes: $20 \mathrm{~V}$ for LPE and PE, $25 \mathrm{~V}$ for LPA, DG, and TG, $30 \mathrm{~V}$ for LPC and LPG, $35 \mathrm{~V}$ for PC and Cer, $40 \mathrm{~V}$ for PG, LPI, SM, HexCer, and CL, $45 \mathrm{~V}$ for Hex2Cer, and $50 \mathrm{~V}$ for PI. The amount of individual lipid species was determined by calculating the corrected peak area which is the ratio of peak area of a lipid species to the area of IS specific to each lipid class. The lipid classes of PC, PE, and TG were quantified by the total numbers of acyl chain and the double bonds because SRM quantification cannot distinguish the isomeric structures while their exact chain locations were identified in the non-targeted identification. All other lipids were quantified by the differences in chain location with each number of the double bond.

\section{Statistical analysis}

Results of biochemical analyses presented as mean with standard error were compared among groups using linear mixed models for repeated measurement. For lipidomic variables, data of each time point after the operation are presented as fold change compared to those of PO. In volcano plots, $\mathrm{P}$ values of lipid species were calculated using paired Student $t$-test or Wilcoxon signed-rank test as appropriate depending on the normality of data distribution. Comparison of each lipid class and species among three 
A

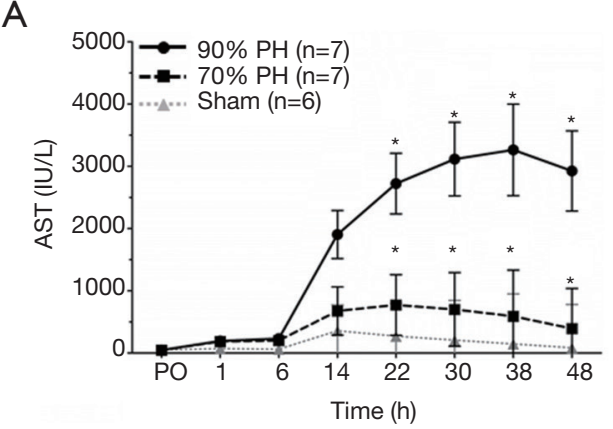

C

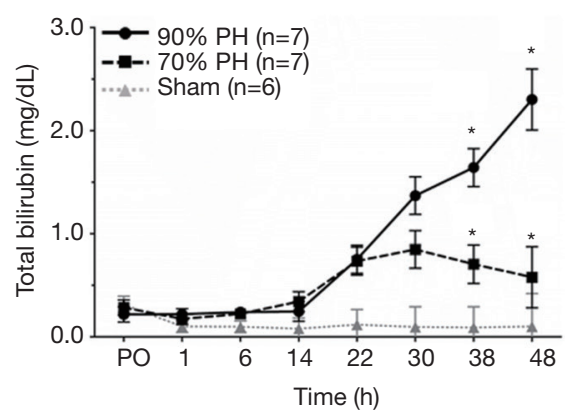

B

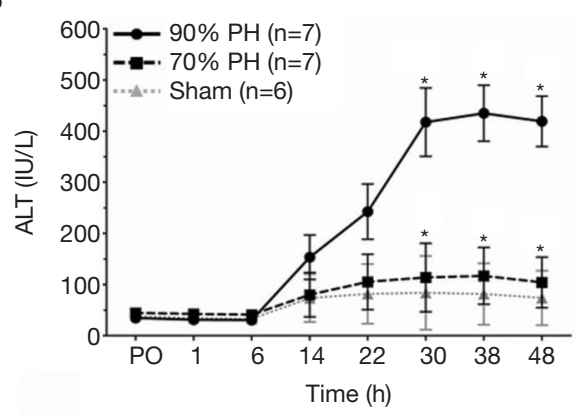

D

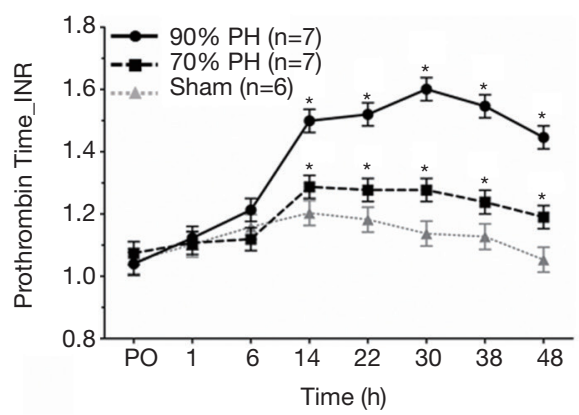

Figure 1 Postoperative evolutions of AST (A), ALT (B), TBIL (C), and PT-INR (D) among sham and each resection group. AST and ALT of the $90 \% \mathrm{PH}$ group showed significantly higher values after 22 and $30 \mathrm{~h}$, respectively, suggesting ongoing hepatocyte injury when compared to the $70 \% \mathrm{PH}$ group. TBIL in the $90 \% \mathrm{PH}$ group showed a tendency to increase above $1 \mathrm{mg} / \mathrm{dL}$ starting from $30 \mathrm{~h}$ after surgery and continued to increase until the time of sacrifice while that in the $70 \% \mathrm{PH}$ group was almost normalized by the time of the sacrifice. PTINR in the $90 \% \mathrm{PH}$ group increased to around the level of 1.5 starting from $14 \mathrm{~h}$ after surgery and peaked at $30 \mathrm{~h}$. In contrast, that of $70 \%$ $\mathrm{PH}$ group peaked around the level of 1.2 at $14 \mathrm{~h}$ after surgery, then followed the recovery trend observed in the sham group. Dots indicate the means, and whiskers indicate the standard error of the means. *, $\mathrm{P}<0.05$ between $70 \% \mathrm{PH}$ and $90 \% \mathrm{PH}$. PH, partial hepatectomy; PO, pre-operation; AST, aspartate aminotransferase; ALT, alanine aminotransferase; TBIL, total bilirubin; PT-INR, prothrombin time as international normalized ratio. Adapted from "Yoon KC, Do Kwon H, Jo HS, et al. Explorative study of serum biomarkers of liver failure after liver resection. Sci Rep 2020;10:9960.” Permission of reprint was waived according to the copyright policy of Scientific Reports.

groups were performed using Kruskal-Wallis test followed by Mann-Whitney U test with Bonferroni correction. The ROC analysis was performed and Pearson's correlation coefficient was calculated for selected lipid species. Minitab 17 statistical software (http://www.minitab.com) was used for the principal component analysis and SPSS software (version 20.0, IBM Corp., Armonk, NY, USA) was used for all other statistical analyses. A $\mathrm{P}$ value $<0.05$ was considered significant, if not indicated otherwise.

\section{Results}

\section{Biochemical analysis after liver resection}

Serial changes of AST, ALT, TBIL, and PT-INR observed in three groups at PO, 1, 6, 14, 22, 30, 38, and $48 \mathrm{~h}$ after surgery are shown in Figure 1. All parameters in the sham group naturally maintained within the normal range at all time points. AST level at 22, 30, 38, $48 \mathrm{~h}$ and ALT level at $30,38,48 \mathrm{~h}$ after surgery were significantly higher in the $90 \% \mathrm{PH}$ group than in the $70 \% \mathrm{PH}$ group $(\mathrm{P}=0.015$, $\mathrm{P}=0.013, \mathrm{P}=0.031, \mathrm{P}=0.017$, and $\mathrm{P}=0.005, \mathrm{P}<0.001, \mathrm{P}<0.001$, respectively). In terms of TBIL and PT-INR which are currently used to diagnose PHLF in a clinical setting, TBIL in the $90 \% \mathrm{PH}$ group constantly increased from $14 \mathrm{~h}$ after surgery until the time of sacrifice and showed higher level at 38 and $48 \mathrm{~h}$ compared to $70 \% \mathrm{PH}$ group $(\mathrm{P}=0.001$, and $\mathrm{P}=0.002$, respectively). PT-INR from $14 \mathrm{~h}$ after surgery was significantly higher in the $90 \% \mathrm{PH}$ group than in the $70 \% \mathrm{PH}$ group $(\mathrm{P}=0.004, \mathrm{P}=0.002, \mathrm{P}<0.001, \mathrm{P}<0.001$, and $\mathrm{P}<0.001$, respectively). 


\section{Global identification of lipids and alterations in lipid levels upon extent of liver resection}

Performance of nUHPLC-ESI-MS/MS runs used for the non-targeted lipid identification of serum lipids is illustrated in Figure S1 with the base peak chromatograms of standard lipids obtained at both positive and negative ion modes of MS detection. The average peak width and the average relative standard deviation in the retention time of lipid standards are $16.2 \pm 7.7 \mathrm{~s}$ and $0.4 \% \pm 0.5 \%$, respectively, along with LOD in the range of 0.006 (LPG 17:1)-0.097 pmol (SM d18:1/17:0), which was estimated from the calibration curves of standards spiked into the serum samples. LOD and limit of quantitation values for other lipid classes are listed in Table S2. For non-targeted lipid identification, a pooled serum sample from each group was utilized (Figure S2) and a total of 284 lipids were identified with their molecular structures. Among them, 184 lipids were quantified by SRM method as the quantification of lipids belonging to PC, PE, TG, and CL was carried out using the total number of acyl chains and double bonds. Quantification was accomplished with individual samples collected at different time points.

Changes in the lipid levels in each group at $30 \mathrm{~h}$ and $48 \mathrm{~h}$ after operation are illustrated in Figure 2 using the Volcano plots. The plots show the decreasing trends in many lipids at 30 and $48 \mathrm{~h}$ after operation when compared to PO. Apparently, more species in the $90 \% \mathrm{PH}$ group showed decreased level as compared to the $70 \% \mathrm{PH}$ and sham groups. Data for all quantified lipid levels are listed in Table S3 and the isomeric chain structures of PC, PE, and TG species are listed in Table S4.

Overall difference in the lipid profiles at different time points after the resection was investigated with principal component analysis plot (Figure 3). While the data points of the $70 \% \mathrm{PH}$ at $30 \mathrm{~h}$ are clustered away from those at 14 $\mathrm{h}$, those at $48 \mathrm{~h}$ are located between the clustered points for 14 and $30 \mathrm{~h}$. However, data points of the $90 \% \mathrm{PH}$ at three different time points are clustered nearby, demonstrating that the changes in the lipid profiles were not distinctive at different time points. This is clearly different from the pattern observed for the $70 \% \mathrm{PH}$ group, implying that changes in the lipid profiles are correlated with the recovery of the liver function upon the degree of resection. Moreover, variations in the lipid profiles of individual animals after resection were not as large as those observed within the sham group, representing that the influence of liver resection on the lipid profiles is much larger than individual differences in the recovery capacity upon the sham surgery.

\section{Alterations in lipid classes}

Alterations in the level of each lipid class at different time points are displayed in Figure 4. Among 16 lipid classes, three classes (PE, PC plasmalogen, and $\mathrm{TG}$ ) shown in Figure $4 A$ exhibited a significant difference ( $>2$-fold and $\mathrm{P}<0.05)$ at $30 \mathrm{~h}$ between the two resection groups. Level of PC plasmalogen was significantly increased in the $90 \%$ $\mathrm{PH}$ group, while those of PE and TG were increased in the $70 \% \mathrm{PH}$ group at $30 \mathrm{~h}$. On the other hand, four classes (LPC, LPE, LPA, and LPI) indicated in Figure $4 B$ exhibited no large difference between the two resection groups until $30 \mathrm{~h}$ but showed a significant difference at $48 \mathrm{~h}$. Lipid levels of these four classes in both resection groups showed a common trend in which they appeared to decrease at $14 \mathrm{~h}$ and tended to recover at $30 \mathrm{~h}$; however, these two resection groups showed an opposite trend at $48 \mathrm{~h}$ in which the 90\% $\mathrm{PH}$ group failed to recover while the $70 \% \mathrm{PH}$ group appeared to recover continuously. Thus, these four lipid classes can be potential candidates for distinguishing the recovery status after $48 \mathrm{~h}$. The sham group without the liver resection showed more than 50\% increases in LPC, LPE, and LPI levels at $14 \mathrm{~h}$ but then decreased to the level of PO, indicating that the increased production of these lipid classes from the liver may indicate a recovery from injury induced by anesthesia, opening, and closure. The remaining nine lipid classes (LPG, PG, PC, PI, DG, SM, Cer, HexCer, and CL) did not show significant changes between the two resection groups as illustrated in Figure S3.

\section{Alterations in the individual lipids}

Alterations in the individual lipid levels can be viewed by the Z-score heat map based on lipid species with significant changes ( $>2$-fold and $\mathrm{P}<0.05$ ) between the two resection groups in Figure 5. Especially, lipid species in PC, PC plasmalogens and TG exhibited striking differences between the two resection groups.

The data were further screened in two ways to select lipid species which showed a significant change over time. First, we selected lipid species which changed more than 2-fold with $\mathrm{P}<0.01$ between the two resection groups both at 30 or $48 \mathrm{~h}$ after the operation. From 184 quantified lipids species, 12 lipid species showed significant differences between the two groups from $30 \mathrm{~h}$; however, only 9 among them 
A Sham
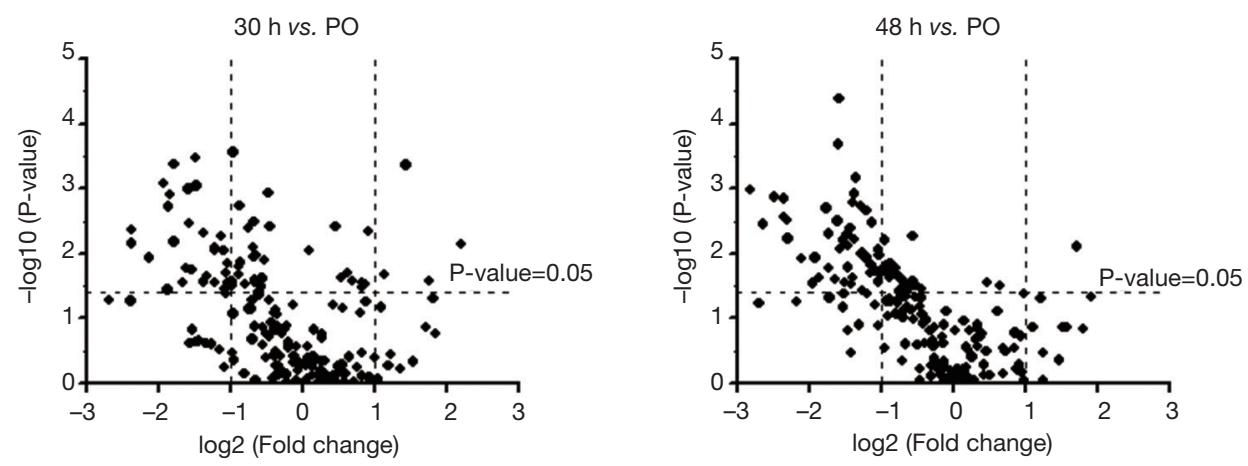

B

$70 \% \mathrm{PH}$
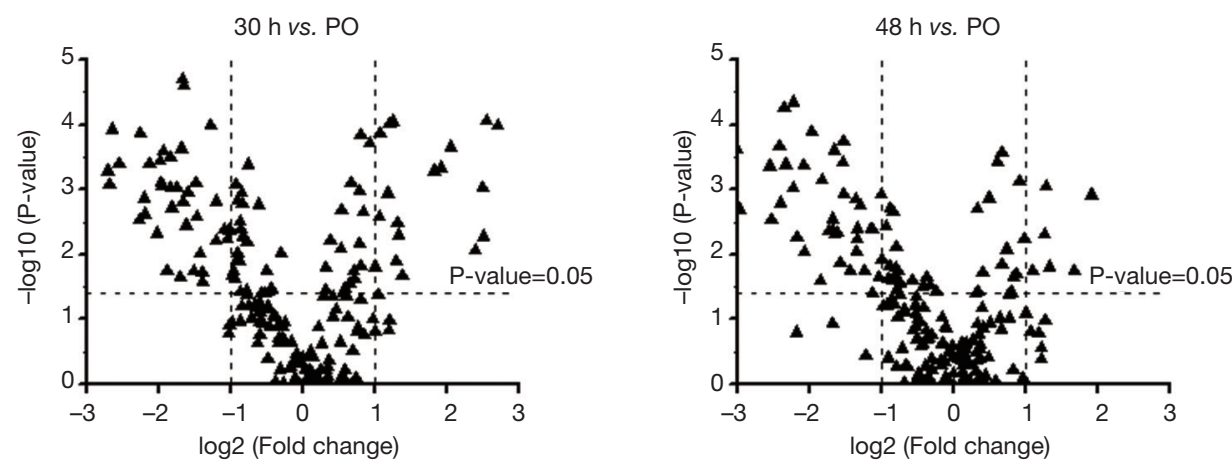

C $\quad 90 \% \mathrm{PH}$
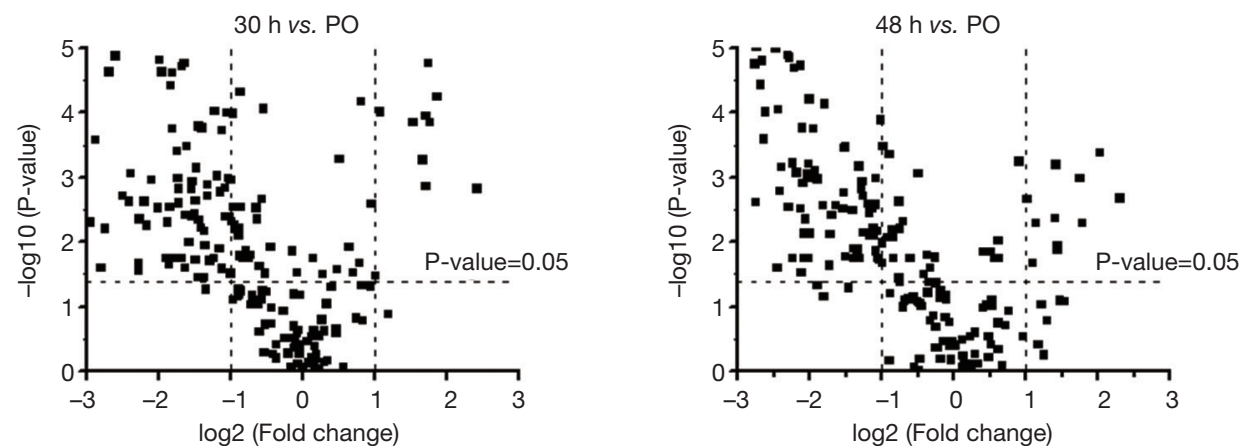

Figure 2 Volcano plot based on fold ratio (vs. PO) of each lipid species at 30 and $48 \mathrm{~h}$ in (A) the sham, (B) the $70 \% \mathrm{PH}$, and (C) the $90 \% \mathrm{PH}$ groups. Data are presented as the $\log 2$ (fold change of individual lipid) on the $\mathrm{x}$ axis and the $-\log 10$ (P value) on the $\mathrm{y}$ axis. The horizontal dashed lines represent the $\mathrm{P}$ value at 0.05 , and the vertical dotted lines represent the 2-fold decrease (A,B,C) and increase. Data points in the left upper domain of each plot represent for lipids that were decreased by more than 2-fold with $\mathrm{P}<0.05$, and those in the right upper domain represent for lipids that were increased by more than 2 -fold with $\mathrm{P}<0.05$. The plots show the decreasing trends in many lipids at 30 and $48 \mathrm{~h}$ after liver resection when compared to PO. Apparently, the level decreased for more species in the $90 \% \mathrm{PH}$ group as compared to the $70 \% \mathrm{PH}$ and sham groups. $\mathrm{PO}$, pre-operation; $\mathrm{PH}$, partial hepatectomy. 


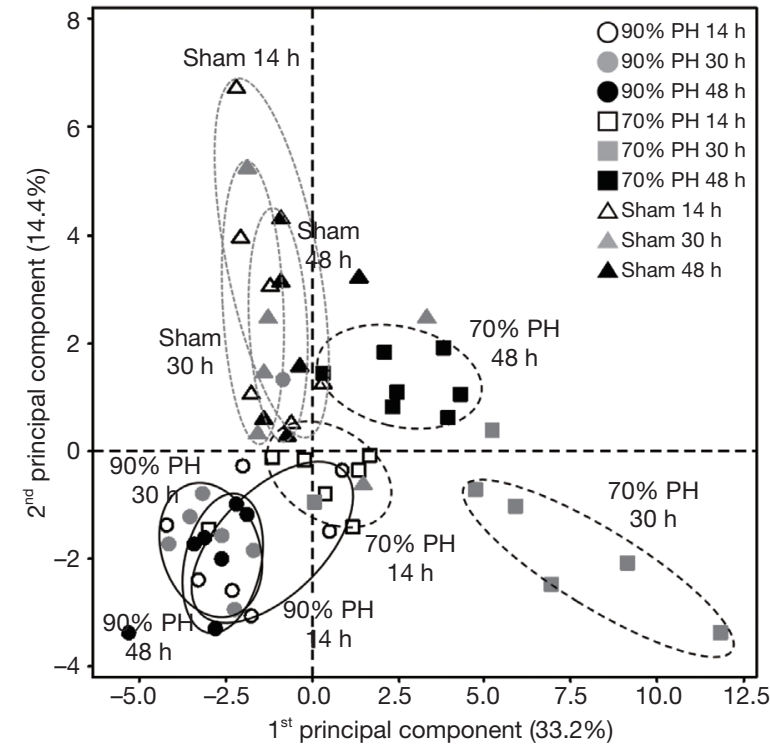

Figure 3 Principal component analysis plots showing the differences in the lipid profiles of the sham, $70 \% \mathrm{PH}$, and $90 \%$ $\mathrm{PH}$ groups at 14,30 , and $48 \mathrm{~h}$ after operation. Plots were based on lipid species showing significant changes $(>2$-fold and $\mathrm{P}<0.05$ ) either in the $70 \% \mathrm{PH}$ or the $90 \% \mathrm{PH}$ group at 30 or $48 \mathrm{~h}$ after the operation. Each point in the plot represents the whole lipid profile of each animal at the specified time point. Data points in three experimental groups are showing distinctive clustering patterns with time point, respectively. Apparently, data points of the $70 \%$ $\mathrm{PH}$ group at 14,30 , and $48 \mathrm{~h}$ are clustered away from each other, indicating that the lipid profiles changed significantly after liver resection over time. However, data points of $90 \% \mathrm{PH}$ group are clustered away from those of both sham and 70\% $\mathrm{PH}$ group without significant change over time. $\mathrm{PH}$, partial hepatectomy.

maintained the difference at $48 \mathrm{~h}$ as shown in Figure $6 \mathrm{~A}$. While the four PC plasmalogens (P-16:0/16:0, P-18:0/18:2, $\mathrm{P}-18: 0 / 20: 4$, and $\mathrm{P}-18: 0 / 22: 6)$ continuously increased until $30 \mathrm{~h}$ in the $90 \% \mathrm{PH}$ group, their levels in the $70 \% \mathrm{PH}$ group increased to some degree at $14 \mathrm{~h}$ but returned to the level similar to those at PO after $30 \mathrm{~h}$. As expected from Figure 4, five TG species (40:0. 42:0. 44:1, 44:2, and 48:3) continuously decreased after $90 \% \mathrm{PH}$, however, their levels in $70 \% \mathrm{PH}$ were increased abruptly to a larger degree (4 6 folds) at $30 \mathrm{~h}$ followed by decreases at $48 \mathrm{~h}$; the levels at 48 $\mathrm{h}$ were nonetheless much higher than those observed for extended resection. TG and PC plasmalogen exhibited an opposite trend between the two resection groups. The acyl chain structures of these TG species are relatively saturated or monounsaturated short chains composed of 14 and 16 carbons.

Next, we screened lipid species showing significant $(\mathrm{P}<0.05)$ differences between the two groups either from 14 or $30 \mathrm{~h}$ and maintaining significant differences thereafter; this selection yielded 14 lipid species. When these were compared with the lipid lists selected in the previous steps, all nine lipid species in Figure $6 A$ were found to have been re-selected. The fold change plots of the five additional species (PC 32:2, TG 42:1, 46:1, 46:2, and SM d18:1/20:0) are displayed in Figure $6 B$.

\section{Receiver operating characteristic (ROC) analysis and the linear correlation}

To investigate the usefulness of the selected 14 lipid species showing significant differences as candidate markers, the ROC analysis was performed. In addition, Pearson correlation coefficient ( $\mathrm{r}$ ) was calculated to find out the relationship between each lipid species at $30 \mathrm{~h}$ and TBIL level or PT-INR. The resulting area under curve (AUC) values of ROC analysis and Pearson correlation coefficient (r) are listed in Table 1. When individual lipid species were analyzed using ROC analysis, their AUC values were larger than 0.900 for all the lipid candidates. On the other hand, calculation of Pearson correlation coefficient ( $r$ ) for each lipid species in combination with TBIL and PT-INR, respectively, showed that all 4 PC plasmalogens were found to be highly correlated $(r>0.7)$ with, both, TBIL and PT-INR.

\section{Changes in PC and LPC species depending on the extent of resection}

Since LPC is produced from PC via lecithin-cholesterol acyltransferase (LCAT) (23), decrease in LPC can be attributed to the reduced activity of LCAT, the production of which in the liver was reduced upon resection. Thus, extended liver resection may result in the substantial decrease in LPC level. To investigate the change in LPC levels in accordance with the corresponding PC molecule, the fold-change plot of PC 32:2, which was contributed from three PC molecules of different acyl chain combinations (PC 14:0/18:2, PC 16:0/16:2, and PC 16:1/16:1) was compared with those of the three possible LPC products (LPC 14:0, LPC 16:0, and LPC 16:1) in Figure 7. While PC 32:2 levels decreased at 30 and $40 \mathrm{~h}$ in the $70 \% \mathrm{PH}$ group, those of the $90 \% \mathrm{PH}$ group were not decreased, but were instead increased slightly. Levels of the 

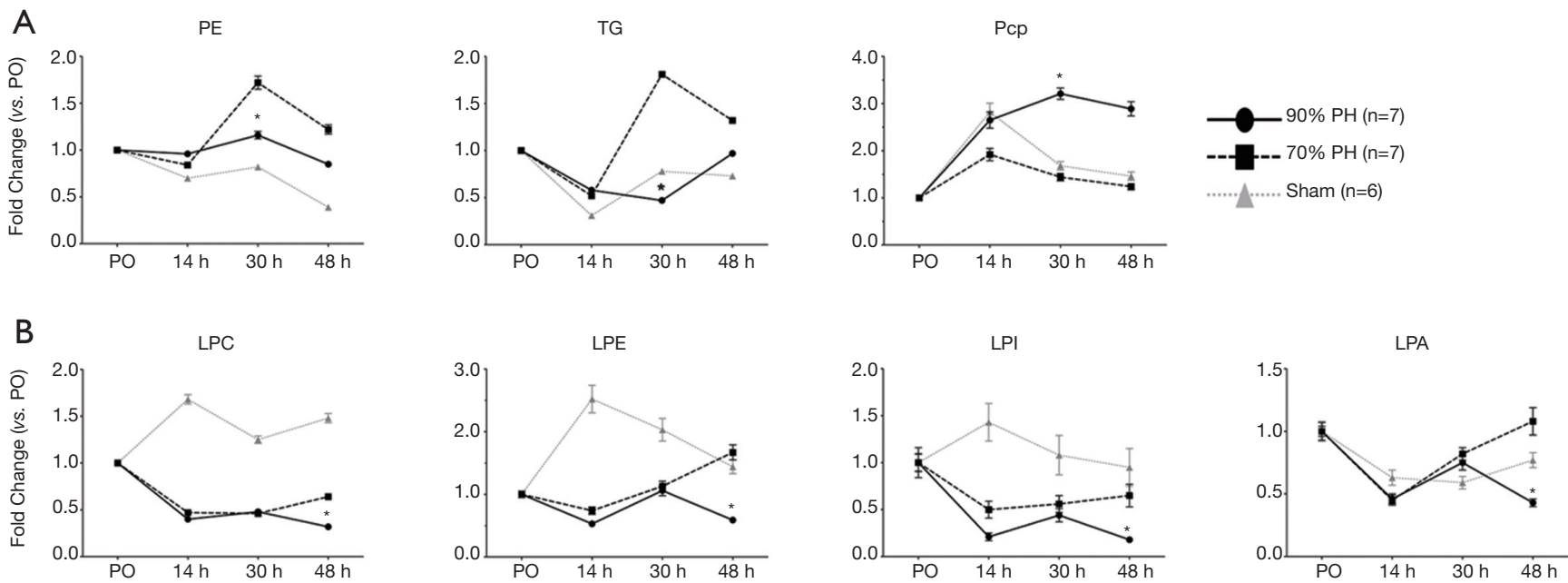

Figure 4 Plots of the fold changes (vs. PO) in lipid classes showing significant changes between the $70 \% \mathrm{PH}$ and $90 \% \mathrm{PH}$ groups (A) at $30 \mathrm{~h}$ and (B) at $48 \mathrm{~h}$ after the operation. Dots indicate the means, and whiskers indicate the standard error of the means. *, $>2$-fold and $\mathrm{P}<0.05$ vs. $70 \% \mathrm{PH}$ group in the same time point. PO, pre-operation; PH, partial hepatectomy; PE, phosphatidylethanolamine; TG, triacylglycerol; PCp, phosphatidylcholine plasmalogen; LPC, lysophosphatidylcholine; LPE, lysophosphatidylethanolamine; LPI, lysophosphatidylinositol; LPA, lysophosphatidic acid.
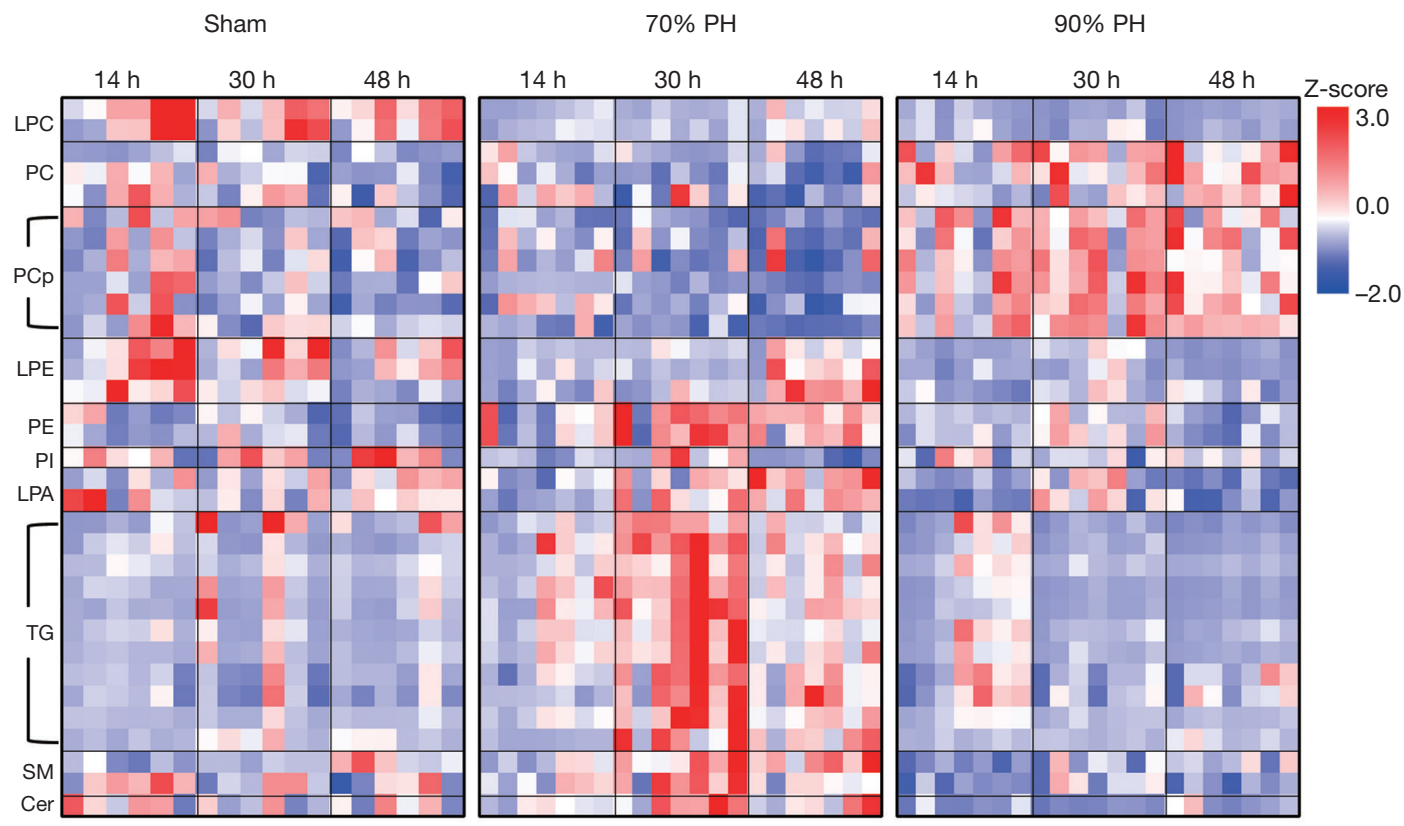

Figure $5 \mathrm{Z}$-score heat map of lipid species showing significant fold-changes ( $v$ s. $\mathrm{PO},>2$-fold and $\mathrm{P}<0.05$ ) between the $70 \% \mathrm{PH}$ and the $90 \%$ $\mathrm{PH}$ at 30 or $48 \mathrm{~h}$ after the operation. Each row represents individual lipid species belonged to lipid class as indicated. Each column indicates the Z-score of lipids obtained from each pig at specified time point. Red color indicates increase in the level of lipid compared to that of PO, whereas blue color indicates decrease. Overall, lipid species in PC and PCp showed increase in 90\% PH group, however, lipid species in the other classes showed increase in $70 \% \mathrm{PH}$ group. The most striking differences between the two resection groups can be observed in lipid species of PC, PCp and TG. PO, pre-operation; PH, partial hepatectomy, LPC, lysophosphatidylcholine; PC, phosphatidylcholine; PCp, phosphatidylcholine plasmalogen; LPE, lysophosphatidylethanolamine; PE, phosphatidylethanolamine; PI, phosphatidylinositol; LPA, lysophosphatidic acid; TG, triacylglycerol; SM, sphingomyelin; Cer, ceramide. 

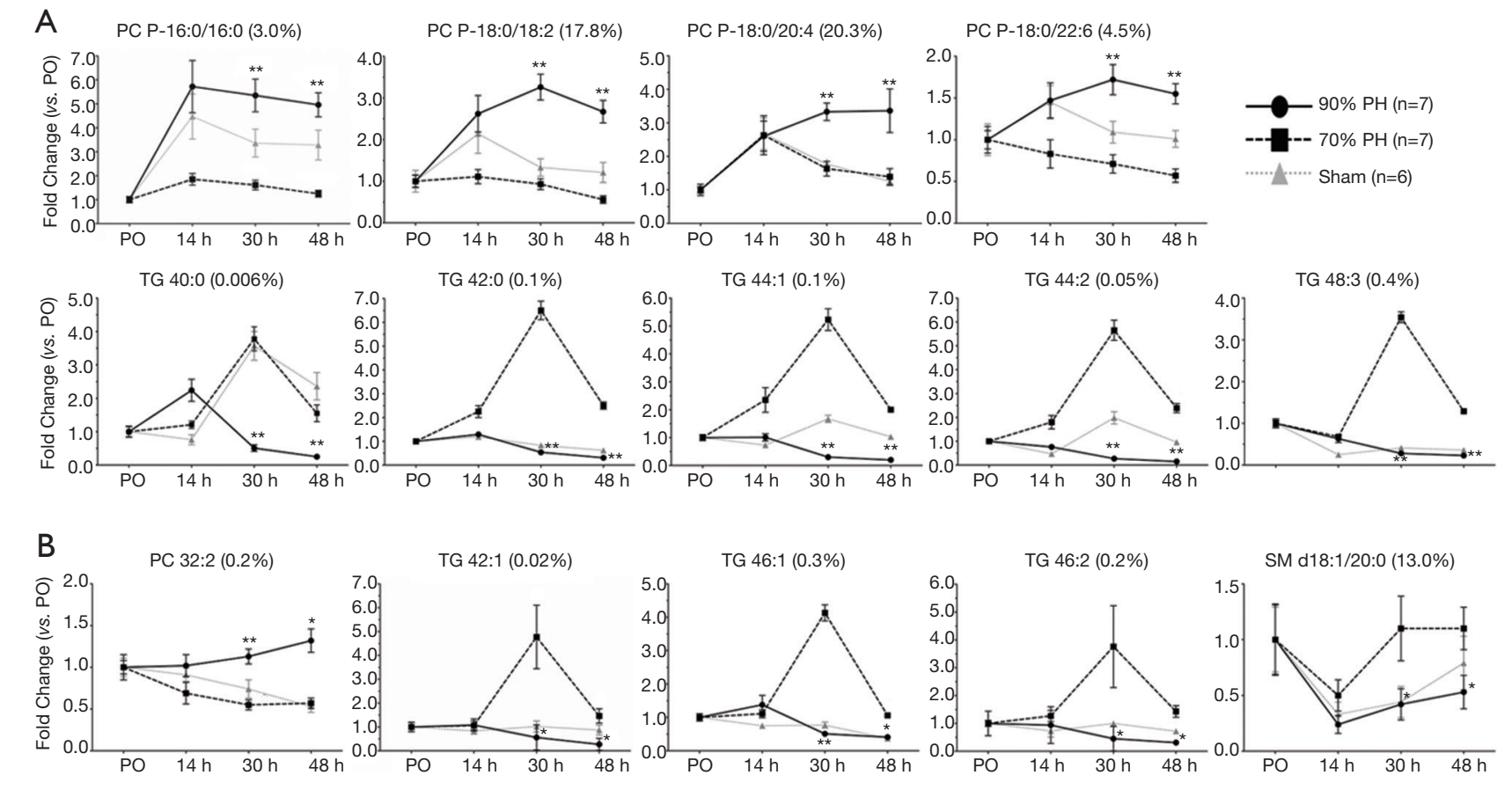

Figure 6 Fold changes (vs. PO) of 14 among 184 quantified lipid species showing (A) significant differences between the $70 \%$ PH and 90\% PH groups at both 30 and $48 \mathrm{~h}$ after the operation, and (B) significant differences between the two resection groups either from 14 or $30 \mathrm{~h}$ and maintaining the significance thereafter, which are not included in (A). The percentages in parentheses next to the name of each lipid species represent the abundance proportion in each lipid class. PC plasmalogens and TGs exhibited an opposite trend between the two resection groups. Lipid levels of four PC plasmalogens increased at $14 \mathrm{~h}$ in both $70 \% \mathrm{PH}$ and $90 \% \mathrm{PH}$ groups. However, those in the $70 \% \mathrm{PH}$ group returned to the level similar to those at $\mathrm{PO}$ after $30 \mathrm{~h}$ while those in the $90 \% \mathrm{PH}$ group continued to increase or maintained at higher level compared to the $70 \% \mathrm{PH}$ group. On the other hand, eight TG species continuously decreased in the $90 \% \mathrm{PH}$ group while those in the $70 \% \mathrm{PH}$ group increased abruptly to a larger degree (4-6 folds) at $30 \mathrm{~h}$ followed by decreases at $48 \mathrm{~h}$; the levels at $48 \mathrm{~h}$ were nonetheless much higher than those observed in the $90 \% \mathrm{PH}$ group. Dots indicate the means, and whiskers indicate the standard error of the means. *, $>2$-fold with $\mathrm{P}<0.01$; **, $\mathrm{P}<0.05$ vs. 70\% $\mathrm{PH}$ group in the same time point. PO, pre-operation; $\mathrm{PH}$, partial hepatectomy; $\mathrm{PC}$, phosphatidylcholine; TG, triacylglycerol, PC P, phosphatidylcholine plasmalogen; SM, sphingomyelin.

three LPC species showed a recovering trend after $30 \mathrm{~h}$ for the $70 \% \mathrm{PH}$ group but those observed with the extended resection were decreased at $48 \mathrm{~h}$. This may support the hypothesis that the reduced production of LPC species is attributed to the deficiency of LCAT upon the failure of the liver function after extended resection.

\section{Discussion}

Considering the clinical significance of PHLF in surgical treatments of various liver diseases, current diagnostic methods have clear limitations with the requirement of better biomarkers to enable earlier diagnosis and development of potential treatments. An important hurdle in investigating PHLF in a clinical setting is difficulties in obtaining well-controlled investigational materials from patients in a reasonable time-frame. In contrast, various porcine models of hepatectomy for the study of liver failure have been developed (24-26), which can provide reasonable alternative research materials for this grave condition. Although a recent study reported a serum metabolic biomarker to predict mortality risk associated with extensive hepatectomy (27), lipidomic analysis to evaluate the time-dependent changes in the individual lipid levels using a PHLF model has not yet been investigated. We employed two $\mathrm{PH}$ models that have a different extent of liver resection. The $70 \% \mathrm{PH}$ model was used as a normal recovery group after massive hepatectomy, considering that $30 \%$ of remnant liver volume is accepted to recover with its regenerative capacity (28). On the other hand, the $90 \%$ 
Table 1 AUC of ROC analysis for the levels of lipid species selected as characteristic signatures to differentiate post-hepatectomy hepatic failure between $70 \% \mathrm{PH}$ and $90 \% \mathrm{PH}$ at 30 hours after the operation. Pearson correlation coefficient, r, value of each individual lipid was calculated in correlation with TBIL and PT-INR levels, respectively

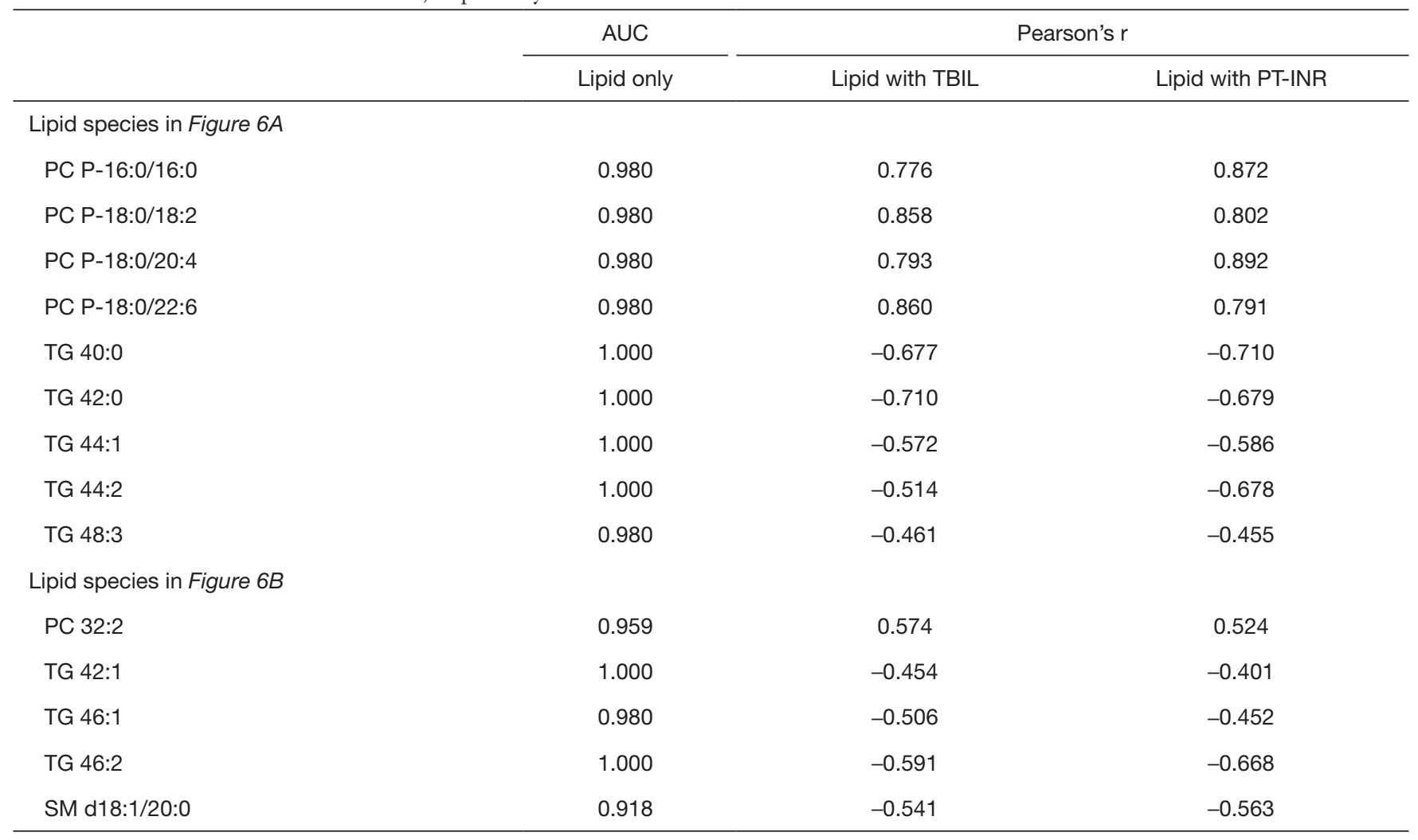

Species marked with bold represent significant correlations ( $r>0.7$ or $<-0.7)$ with both TBIL and PT-INR. AUC, area under curve; ROC, receiver operating characteristic; $\mathrm{PH}$, partial hepatectomy; TBIL, total bilirubin; PT-INR, prothrombin time as international normalized ratio; PC P, phosphatidylcholine plasmalogen; TG, triacylglycerol; SM, sphingomyelin.
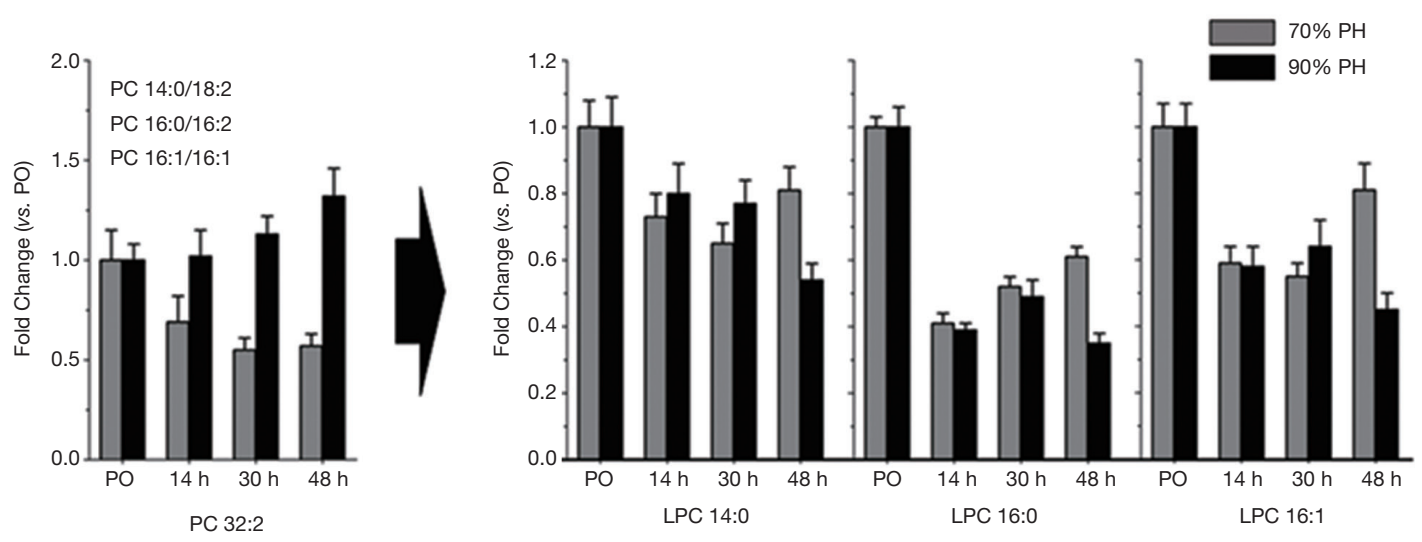

Figure 7 Fold changes (vs. PO) of PC 32:2 and three LPC species (14:0, 16:0, and 16:1) showing difference with $\mathrm{P}<0.01$ between the 70\% $\mathrm{PH}$ and $90 \% \mathrm{PH}$ group to investigate the change in LPC levels in accordance with the corresponding PC molecule. Those LPC species have the common acyl chain contained in these PC species. The level of PC 32:2 decreased in the $70 \% \mathrm{PH}$ group at 30 and $48 \mathrm{~h}$ while that in the $90 \% \mathrm{PH}$ group increased slightly. The levels of three LPC species in the $70 \% \mathrm{PH}$ group decreased after the operation and recovered after $30 \mathrm{~h}$, but those in the $90 \% \mathrm{PH}$ group did not recover until the time of sacrifice. PO, pre-operation; LPC, lysophosphatidylcholine; PH, partial hepatectomy; PC, phosphatidylcholine. 
$\mathrm{PH}$ model was regarded as a clinically relevant model to induce PHLF (29). Previous studies have reported that all animals in their PHLF model died before $51 \mathrm{~h}$ following hepatectomy (30). Therefore, end-point in the present experiment was set as $48 \mathrm{~h}$ following hepatectomy. The adequacy of experimental models used in this study is well supported by Figure 1. AST and ALT are present in the hepatocytes, and these enzymes leak into the serum when hepatocytes are damaged in various conditions (31). Therefore, higher AST and ALT levels in the 90\% PH group indicated more ongoing hepatocyte injury compared to the $70 \% \mathrm{PH}$ group. TBIL and PT-INR reflecting excretory and synthetic function of the liver (2) dramatically increased in the $90 \% \mathrm{PH}$ group or showed returning trends toward baseline level in the $70 \% \mathrm{PH}$ group over time. These four biochemical parameters are widely used to assess the functional status of the liver in a clinical setting.

The use of nUHPLC-ESI-MS/MS for lipid analysis greatly enhanced the resolution of lipid separation with the LOD at low femtomole levels as well as facilitated high speed, targeted quantification of several hundred lipids with only a few micrograms of lipid sample $(20,21)$. Lipid species selected in Figure 6 can be classified into two subgroups. Four PC plasmalogens and PC 32:2, which showed significantly increased level in $90 \% \mathrm{PH}$ group reflecting severity of injury or inflammation, can be considered as failure marker group. In contrast, 8 TGs and SM d18:1/20:0, which showed significant increase in $70 \%$ $\mathrm{PH}$ group and return to baseline by $48 \mathrm{~h}$, can be considered as recovery marker group. Interestingly, the serial changes in lipid class of PC plasmalogen and TG showed the same trend as selected individual species (Figure 4A).

Plasmalogens are phospholipids that constitute a significant amount of total membrane lipids and are known to protect from oxidative damage (32). Plasmalogens are predominantly synthesized in the liver and excreted into blood stream as lipoproteins. In mammals, plasmalogens are enriched in brain, kidney and heart, however, the lowest amounts of plasmalogen are found in liver (32). The levels of PC plasmalogens between the two $\mathrm{PH}$ groups in this study exhibited clear differences. While PC plasmalogen levels of the $90 \% \mathrm{PH}$ group were continuously elevated with the maximum at or around $30 \mathrm{~h}$ and then were maintained at high levels or slightly decreased, those of the $70 \% \mathrm{PH}$ group were similar to or little lower than that of PO. One possible explanation for this finding is a dysregulated excessive synthesis of plasmalogens by the liver due to very large oxidative stress after excessive liver resection. Liver resection induces inflammatory response and oxidative stress, which may consume the antioxidant capacity to maintain the oxidative stress within certain range during normal recovery (33), as observed in the $70 \% \mathrm{PH}$ group. However, if the extent of liver resection exceeds recoverable range facing excessive oxidative stress, the homeostasis of plasmalogen biosynthesis might be dysregulated, resulting in an abnormally high level of plasmalogens in the serum. Another potential mechanism is that the high plasmalogens levels in the serum of the $90 \% \mathrm{PH}$ group might originate from injury in remote organs inflicted by acute liver failure after extensive liver resection. It is well known that kidney and brain can exhibit remote organ injury from acute liver failure (34). Though the plasmalogen level in the liver is very low, the serum levels of plasmalogen in patients with mild liver inflammation or dysfunction such as nonalcoholic steatohepatitis were significantly decreased in comparison to those of healthy controls, which could be due to peroxisomal dysfunction and/or impaired hepatic biosynthesis of plasmalogens (35). In contrast, kidney and brain contain high levels of plasmalogen and are easily injured in the setting of acute liver failure suggesting that high levels of plasmalogen in the serum of the $90 \% \mathrm{PH}$ group could originate from the remote injury in kidney or brain.

It has long been recognized that the regenerating liver generates signals that couple release of fatty acids from peripheral adipose stores to augment the hepatic fatty acid uptake and leads to rapid accumulation of intracellular TG (15,36-38); nonetheless, there is a controversy regarding an increase in the secretion of TG into the blood during regeneration $(36,39)$. TG is also known to be regulated by bilirubin (40). Studies have shown that bilirubin decreased the serum levels of total cholesterol and TG as it suppressed the expression of few protein factors involved in TG synthesis, which were elevated in the rat livers with diabetes (40). In the present study, the levels of most TGs showed an opposite trend at $30 \mathrm{~h}$ between $70 \%$ and $90 \% \mathrm{PH}$ groups, suggesting that there were significant differences in TG metabolism and trafficking between the two groups; however, it remains unclear whether these contrasts were due to the difference in the capacity of liver regeneration or due to that of serum bilirubin levels at this time point. However, TG levels of the $90 \% \mathrm{PH}$ group at $48 \mathrm{~h}$ dropped down significantly to make the difference between the two groups less significant than that at $30 \mathrm{~h}$, suggesting less predictive power. Nevertheless, TG can be recognized as a recovery marker after the liver resection while PC plasmalogen can be considered as a failure 
marker, provided PHLF occurs with the $90 \% \mathrm{PH}$ group.

LPC levels were reported to decrease in the rat serum and liver after extended liver resection (19), which is similar to the observed decrease in this study (Figure 4). On the other hand, although the overall PC levels of the two resection groups were found to decrease in this study (Figure S3) similar to other report (19), that of PC 32:2 in $90 \% \mathrm{PH}$ group was increased. Decrease in serum LPC levels was reported to be attributed to the resection-induced impairment of the liver, thus, reducing the production of albumin, which transports LPC through blood (41). Decrease in LPC can originate from the reduced activity of LCAT, which is accompanied by increase in certain PC species. Due to the decrease in the liver function upon resection, synthesis of LCAT in the liver is reduced, resulting in the decreased production of LPC. This may imply that the recovery in LPC levels at $48 \mathrm{~h}$ in the $70 \%$ $\mathrm{PH}$ group and the continuous decrease in the extended resection group strongly support the recovery and the failure of the liver function, respectively (Figure 7). Moreover, LCAT levels in patients with liver disease were reported to decrease upon increase in the bilirubin level (42). In our study, TBIL levels increased to $0.85 \pm 0.81 \mathrm{ng} / \mathrm{dL}(70 \% \mathrm{PH})$ and $1.37 \pm 0.47 \mathrm{ng} / \mathrm{dL}(90 \% \mathrm{PH})$ at $30 \mathrm{~h}$, which was about 3-6 folds higher as compared to those at PO; however, at $48 \mathrm{~h}$, the TBIL level further increased significantly in the extended resection group $(2.30 \pm 1.15 \mathrm{ng} / \mathrm{dL})$ whereas it decreased to $0.58 \pm 0.96 \mathrm{ng} / \mathrm{dL}$ for the $70 \% \mathrm{PH}$. This supports that the increase in the TBIL level at $48 \mathrm{~h}$ caused by the liver damage is correlated with the decrease in LCAT level and further to LPC levels.

Considering both that serum TBIL and PT-INR are the most widely used biochemical parameters reflecting liver function and that they cannot accurately predict PHLF by postoperative day 5 , significant correlation of certain lipid species with those in the early postoperative period does not necessarily imply higher potential as a biomarker. Rather, human-derived studies are warranted to establish a model for predicting PHLF in the early postoperative period by combining the recovery and failure lipid markers identified in this study. Eventually, it may pave the way to overcome PHLF by enabling the rapid application of potential treatments and evaluating their effects.

\section{Conclusions}

Although the present study was conducted with limited number of animals using experimental settings at its extreme, the results revealed the potential of the utilization of lipid species as markers to distinguish the early stage of PHLF after the liver resection, which can be critical for early diagnosis and helpful to develop a timely intervention for the recovery.

\section{Acknowledgments}

Funding: This study was supported by the grants (NRF2017R1A2B2005754 and NRF-2018R1A2A1A05019794) awarded by the Ministry of Science and ICT through the National Research Foundation (NRF) of Korea.

\section{Footnote}

Reporting Checklist: The authors have completed the ARRIVE reporting checklist. Available at http://dx.doi. org/10.21037/atm-20-3596

Data Sharing Statement: Available at http://dx.doi. org/10.21037/atm-20-3596

Peer Review File: Available at http://dx.doi.org/10.21037/ atm-20-3596

Conflicts of Interest: All authors have completed the ICMJE uniform disclosure form (available at http:// dx.doi.org/10.21037/atm-20-3596). DSK reports grants from Ministry of Science and ICT through the National Research Foundation (NRF) of Korea, grants from Ministry of Science and ICT through the National Research Foundation (NRF) of Korea, during the conduct of the study. The other authors have no conflicts of interest to declare.

Ethical Statement: The authors are accountable for all aspects of the work in ensuring that questions related to the accuracy or integrity of any part of the work are appropriately investigated and resolved. This study was approved by the Korea University Institutional Animal Care and Use Committee (Institutional Review Board: KOREA2017-0069-C3) and all animals were cared for in accordance with the National guidelines for ethical animal research.

Open Access Statement: This is an Open Access article distributed in accordance with the Creative Commons Attribution-NonCommercial-NoDerivs 4.0 International License (CC BY-NC-ND 4.0), which permits the non- 
commercial replication and distribution of the article with the strict proviso that no changes or edits are made and the original work is properly cited (including links to both the formal publication through the relevant DOI and the license). See: https://creativecommons.org/licenses/by-nc-nd/4.0/.

\section{References}

1. Rahman SH, Evans J, Toogood GJ, et al. Prognostic utility of postoperative C-reactive protein for posthepatectomy liver failure. Arch Surg 2008;143:247-53.

2. Rahbari NN, Garden OJ, Padbury R, et al. Posthepatectomy liver failure: a definition and grading by the International Study Group of Liver Surgery (ISGLS). Surgery 2011;149:713-24.

3. Ray S, Mehta NN, Golhar A, et al. Post hepatectomy liver failure - A comprehensive review of current concepts and controversies. Ann Med Surg (Lond) 2018;34:4-10.

4. Kauffmann R, Fong Y. Post-hepatectomy liver failure. Hepatobiliary Surg Nutr 2014;3:238-46.

5. Gilg S, Sandstrom P, Rizell M, et al. The impact of posthepatectomy liver failure on mortality: a population-based study. Scand J Gastroenterol 2018;53:1335-9.

6. Lafaro K, Buettner S, Maqsood H, et al. Defining Post Hepatectomy Liver Insufficiency: Where do We stand? J Gastrointest Surg 2015;19:2079-92.

7. Niiya T, Murakami M, Aoki T, et al. Immediate increase of portal pressure, reflecting sinusoidal shear stress, induced liver regeneration after partial hepatectomy. J Hepatobiliary Pancreat Surg 1999;6:275-80.

8. Sato Y, Koyama S, Tsukada K, et al. Acute portal hypertension reflecting shear stress as a trigger of liver regeneration following partial hepatectomy. Surg Today 1997;27:518-26.

9. Balzan S, Belghiti J, Farges O, et al. The "50-50 criteria" on postoperative day 5: an accurate predictor of liver failure and death after hepatectomy. Ann Surg 2005;242:824-8.

10. Wenk MR. The emerging field of lipidomics. Nat Rev Drug Discov 2005;4:594-610.

11. Bosio A, Binczek E, Stoffel W. Functional breakdown of the lipid bilayer of the myelin membrane in central and peripheral nervous system by disrupted galactocerebroside synthesis. Proc Natl Acad Sci U S A 1996;93:13280-5.

12. Nguyen P, Leray V, Diez M, et al. Liver lipid metabolism. J Anim Physiol Anim Nutr (Berl) 2008;92:272-83.

13. Kaiser T, Kinny-Koster B, Bartels M, et al. Cholesterol esterification in plasma as a biomarker for liver function and prediction of mortality. BMC Gastroenterol 2017;17:57.

14. Rudnick DA, Davidson NO. Functional Relationships between Lipid Metabolism and Liver Regeneration. Int J Hepatol 2012;2012:549241.

15. Delahunty TJ, Rubinstein D. Accumulation and release of triglycerides by rat liver following partial hepatectomy. J Lipid Res 1970;11:536-43.

16. Gove CD, Hems DA. Fatty acid synthesis in the regenerating liver of the rat. Biochem J 1978;170:1-8.

17. Walldorf J, Hillebrand C, Aurich H, et al. Propranolol impairs liver regeneration after partial hepatectomy in C57Bl/6-mice by transient attenuation of hepatic lipid accumulation and increased apoptosis. Scand J Gastroenterol 2010;45:468-76.

18. Feldstein AE, Canbay A, Angulo P, et al. Hepatocyte apoptosis and fas expression are prominent features of human nonalcoholic steatohepatitis. Gastroenterology 2003;125:437-43.

19. Tautenhahn HM, Bruckner S, Baumann S, et al. Attenuation of Postoperative Acute Liver Failure by Mesenchymal Stem Cell Treatment Due to Metabolic Implications. Ann Surg 2016;263:546-56.

20. Byeon SK, Lee JC, Chung BC, et al. High-throughput and rapid quantification of lipids by nanoflow UPLCESI-MS/MS: application to the hepatic lipids of rabbits with nonalcoholic fatty liver disease. Anal Bioanal Chem 2016;408:4975-85.

21. Lee JC, Byeon SK, Moon MH. Relative Quantification of Phospholipids Based on Isotope-Labeled Methylation by Nanoflow Ultrahigh Performance Liquid Chromatography-Tandem Mass Spectrometry: Enhancement in Cardiolipin Profiling. Anal Chem 2017;89:4969-77.

22. Lim S, Byeon SK, Lee JY, et al. Computational approach to structural identification of phospholipids using raw mass spectra from nanoflow liquid chromatography-electrospray ionization-tandem mass spectrometry. J Mass Spectrom 2012;47:1004-14.

23. Glomset JA. The mechanism of the plasma cholesterol esterification reaction: plasma fatty acid transferase. Biochim Biophys Acta 1962;65:128-35.

24. Golriz M, El Sakka S, Majlesara A, et al. Hepatic Hemodynamic Changes Following Stepwise Liver Resection. J Gastrointest Surg 2016;20:587-94.

25. Arkadopoulos N, Defterevos G, Nastos C, et al. Development of a porcine model of post-hepatectomy liver failure. J Surg Res 2011;170:e233-42. 
26. Court FG, Laws PE, Morrison CP, et al. Subtotal hepatectomy: a porcine model for the study of liver regeneration. J Surg Res 2004;116:181-6.

27. Yoon KC, Do Kwon H, Jo HS, et al. Explorative study of serum biomarkers of liver failure after liver resection. Sci Rep 2020;10:9960.

28. Ferrero A, Viganò L, Polastri R, et al. Postoperative liver dysfunction and future remnant liver: where is the limit? World J Surg 2007;31:1643-51.

29. Athanasiou A, Spartalis E. Porcine models for the study of small-for-size syndrome. J Hepatobiliary Pancreat Sci 2017;24:E6-7.

30. Chen HS, Joo DJ, Shaheen M, et al. Randomized Trial of Spheroid Reservoir Bioartificial Liver in Porcine Model of Posthepatectomy Liver Failure. Hepatology 2019;69:329-42.

31. Ge PL, Du SD, Mao YL. Advances in preoperative assessment of liver function. Hepatobiliary Pancreat Dis Int 2014;13:361-70.

32. Braverman NE, Moser AB. Functions of plasmalogen lipids in health and disease. Biochim Biophys Acta 2012;1822:1442-52.

33. Schwarz C, Fitschek F, Bar-Or D, et al. Inflammatory response and oxidative stress during liver resection. PLoS One 2017;12:e0185685.

34. Nastos C, Kalimeris K, Papoutsidakis N, et al. Global consequences of liver ischemia/reperfusion injury. Oxid

Cite this article as: Jo HS, Kim HA, Lee JC, Yoon KC, Yoon YI, Choi YY, Seok JI, Moon MH, Kim DS. Lipidomic signatures of post-hepatectomy liver failure using porcine hepatectomy models. Ann Transl Med 2020;8(21):1363. doi: 10.21037/atm-20-3596
Med Cell Longev 2014;2014:906965.

35. Ikuta A, Sakurai T, Nishimukai M, et al. Composition of plasmalogens in serum lipoproteins from patients with non-alcoholic steatohepatitis and their susceptibility to oxidation. Clin Chim Acta 2019;493:1-7.

36. Kaibori M, Kwon AH, Oda M, et al. Hepatocyte growth factor stimulates synthesis of lipids and secretion of lipoproteins in rat hepatocytes. Hepatology 1998;27:1354-61.

37. Farrell GC. Probing Prometheus: fat fueling the fire? Hepatology 2004;40:1252-5.

38. Newberry EP, Kennedy SM, Xie Y, et al. Altered hepatic triglyceride content after partial hepatectomy without impaired liver regeneration in multiple murine genetic models. Hepatology 2008;48:1097-105.

39. Tijburg LB, Nyathi CB, Meijer GW, et al. Biosynthesis and secretion of triacylglycerol in rat liver after partial hepatectomy. Biochem J 1991;277:723-8.

40. Xu J, Lee ES, Baek SH, et al. Effect of bilirubin on triglyceride synthesis in streptozotocin-induced diabetic nephropathy. J Korean Med Sci 2014;29 Suppl 2:S155-63.

41. Baisted DJ, Robinson BS, Vance DE. Albumin stimulates the release of lysophosphatidylcholine from cultured rat hepatocytes. Biochem J 1988;253:693-701.

42. Florén CH, Chen CH, Franzen J, et al. Lecithin: cholesterol acyltransferase in liver disease. Scand J Clin Lab Invest 1987;47:613-7. 